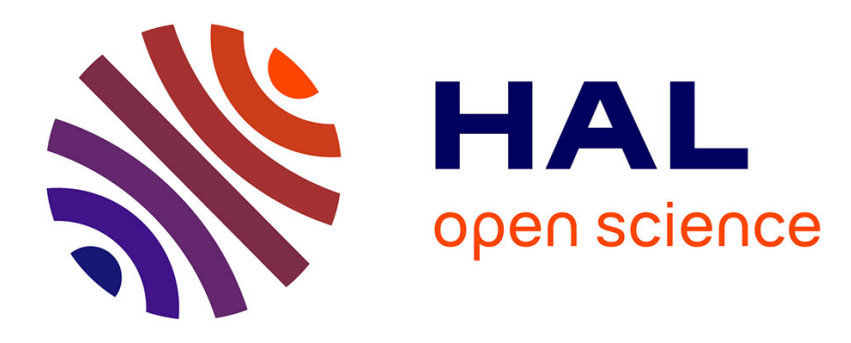

\title{
Orbit Equivalence and Measured Group Theory
}

Damien Gaboriau

\section{To cite this version:}

Damien Gaboriau. Orbit Equivalence and Measured Group Theory. International Congress of Mathematicians (ICM), International Mathematical Union (IMU) / M.S. Raghunathan, Tata Institute of Fundamental Research, Mumbai, 2010, Hyderabad, India. pp.1501-1527. ensl-00512729

\section{HAL Id: ensl-00512729 \\ https://hal-ens-lyon.archives-ouvertes.fr/ensl-00512729}

Submitted on 31 Aug 2010

HAL is a multi-disciplinary open access archive for the deposit and dissemination of scientific research documents, whether they are published or not. The documents may come from teaching and research institutions in France or abroad, or from public or private research centers.
L'archive ouverte pluridisciplinaire HAL, est destinée au dépôt et à la diffusion de documents scientifiques de niveau recherche, publiés ou non, émanant des établissements d'enseignement et de recherche français ou étrangers, des laboratoires publics ou privés. 


\title{
Orbit Equivalence and Measured Group Theory
}

\author{
Damien Gaboriau*
}

May, 2010

\begin{abstract}
We give a survey of various recent developments in orbit equivalence and measured group theory. This subject aims at studying infinite countable groups through their measure preserving actions.
\end{abstract}

2000 Mathematical Subject Classification: Primary 37A20; Secondary 46L10; 28D15. Key words: Orbit equivalence, Measured group theory, von Neumann algebras.

\section{Introduction}

Orbit equivalence and measure equivalence theories deal with countable groups $\Gamma$ acting on standard measure spaces and with the associated orbit partitions of the spaces. This is very much connected from its birth with operator algebras [MvN36]; many of the recent progresses in both areas were made conjointly (see [Pop07b, Vae07, Vae10]). It turns out to be also connected with geometric group theory (see section 9 and [Fur09]), descriptive set theory (see JKL02, KM04]), percolation on graphs (see [LP09])... with fruitful crosspollination.

There are many examples of mathematical domains where the orbit equivalence or measured approach helps solving delicate questions involving countable groups $\Gamma$. For instance, in connection with group $\ell^{2}$-Betti numbers $\beta_{n}^{(2)}(\Gamma)$, this was useful to attack:

- various vanishing results in Gab02, ST07;

- the study of harmonic Dirichlet functions on percolation subgraphs Gab05b;

- the comparison between the uniform isoperimetric constant and $\beta_{1}^{(2)}(\bar{\Gamma})$ [LPV08];

- problems of topological nature, related to the work of Gromov about the minimal volume Sau09.

In geometric group theory, the quasi-isometry invariance of various cohomological properties for amenable groups [Sha04] was obtained that way. Gaboriau-Lyons' measurable solution to von Neumann's problem GL09] happens to be a way to extend results about groups containing a copy of the free group $\mathbf{F}_{2}$ to every non-amenable group (see section 10). This was used by Eps08] and in Dixmier's unitarizability problem [EM09, M010].

${ }^{*}$ CNRS / ANR AGORA NANR-09-BLAN-0059 
The purpose of this survey is to describe some foundations of the theory and some of its most recent developments. There are many aspects upon which we shall inevitably not touch here, and many results are just alluded to, with as far as possible the relevant bibliography.

There are several excellent books and surveys with various focuses on orbit equivalence to which the reader is referred for further information, for instance KM04, Gab05a, Sha05, Pop07b, Fur09, Kec10.

\section{Setting and examples}

The measure spaces $X$ will always be assumed to be standard Borel spaces and unless specified otherwise, the measure $\mu$ will be a non-atomic probability measure. Measurably, $(X, \mu)$ is isomorphic to the interval $([0,1]$, Leb) equipped with the Lebesgue measure. Moreover, the actions $\Gamma \curvearrowright^{\alpha}(X, \mu)$ we shall consider will be by Borel automorphisms and probability measure preserving (p.m.p.), i.e. $\forall \gamma \in \Gamma, A \subset X: \mu(\gamma \cdot A)=\mu(A)$ (We only considers Borel sets). Shortly, $\alpha$ is a p.m.p. action of $\Gamma$. In this measured context, null sets are neglected. Equality for instance is always understood almost everywhere. The action $\alpha$ is (essentially) free if $\mu\{x: \gamma \cdot x=x\}>0 \Rightarrow \gamma=i d$. The action is ergodic if the dynamics is indecomposable, i.e. whenever $X$ admits a partition $X=A \cup{ }^{c} A$ into invariant Borel subsets, then one of them is trivial, i.e. $\mu(A) \mu\left({ }^{c} A\right)=0$.

We now present a series of basic examples which shall already exhibit a rich variety of phenomena.

Examples 2.1 The action of $\mathbb{Z}^{n}$ on the circle $\mathbb{S}^{1}$ by rationally independent rotations.

Examples 2.2 The standard action $\operatorname{SL}(n, \mathbb{Z}) \curvearrowright \mathbb{T}^{n}$ on the $n$-torus $\mathbb{R}^{n} / \mathbb{Z}^{n}$ with the Lebesgue measure. The behavior is drastically different for $n \geq 3$ and for $n=2$. The higher dimensional case was central in the super-rigidity results of Zimmer [im84 and Furman Fur99d, Fur996] (see section 19 ). The 2-dimensional case $\operatorname{SL}(2, \mathbb{Z}) \curvearrowright \mathbb{T}^{2}$ played a particularly important role in the recent developments of the theory, mainly because of its relation with the semi-direct product $\operatorname{SL}(2, \mathbb{Z}) \ltimes \mathbb{Z}^{2}$, in which $\mathbb{Z}^{2}$ is known to have the so called relative property $(T)$ (see section 11 ), while $\mathrm{SL}(2, \mathbb{Z})$ is a virtually free group (it has a finite index free subgroup).

Examples 2.3 Volume-preserving group actions on finite volume manifolds.

Examples 2.4 Given two lattices $\Gamma, \Lambda$ in a Lie group $H$ (or more generally a locally compact second countable group) the actions by left (resp. right by the inverse) multiplication on $H$ induce actions on the finite measure standard spaces $\Gamma \curvearrowright H / \Lambda$ and $\Lambda \curvearrowright \Gamma \backslash H$.

Examples 2.5 A compact group $K$, its Haar measure $\mu$ and the action of a countable subgroup $\Gamma$ by left multiplication on $K$. 
Examples 2.6 Let $\left(X_{0}, \mu_{0}\right)$ be a standard probability measure space, possibly with atoms The standard Bernoulli shift action of $\Gamma$ is the action on the space $X^{\Gamma}$ of sequences $\left(x_{\gamma}\right)_{\gamma \in \Gamma}$ by shifting the indices $g .\left(x_{\gamma}\right)_{\gamma \in \Gamma}=\left(x_{g^{-1} \gamma}\right)_{\gamma \in \Gamma}$, together with the $\Gamma$-invariant product probability measure $\otimes_{\Gamma} \mu_{0}$. In particular, every countable group admits at least one p.m.p. action. The action is free (and ergodic) iff $\Gamma$ is infinite.

More generally, consider some action $\Gamma \curvearrowright V$ of $\Gamma$ on some countable set $V$. The generalized Bernoulli shift action of $\Gamma$ is the action on the space $X^{V}$ of sequences $\left(x_{v}\right)_{v \in V}$ by shifting the indices $g \cdot\left(x_{v}\right)_{v \in V}=\left(x_{g^{-1} . v}\right)_{v \in V}$, with the invariant product probability measure.

Examples 2.7 Profinite actions. Consider an action $\Gamma \curvearrowright\left(\mathrm{T}, v_{0}\right)$ of $\Gamma$ on a locally finite rooted tree. The action preserves the equiprobability on the levels, and the induced limit probability measure on the set of ends of the tree is $\Gamma$-invariant. For instance, if $\Gamma$ is residually finite, as witnessed by a chain of finite index subgroups $\Gamma=\Gamma_{0}>\Gamma_{1}>$ $\Gamma_{2}>\cdots \Gamma_{i}>\cdots$ with trivial intersection, such a rooted tree $\left(\mathrm{T},\left(v_{0}=\Gamma / \Gamma_{0}\right)\right)$ is naturally built with vertex set (of level $i$ ) the cosets $\Gamma / \Gamma_{i}$ and edges given by the reduction maps $\Gamma / \Gamma_{i+1} \rightarrow \Gamma / \Gamma_{i}$. The action is ergodic iff it is transitive on the levels.

A first connection with functional analysis is made through the following. The Koopman representation of a p.m.p. action $\Gamma \curvearrowright^{\alpha}(X, \mu)$ is the representation $\kappa_{\alpha}$ of $\Gamma$ on $L_{0}^{2}(X, \mu)$ given by $\kappa_{\alpha}(\gamma)(\xi)(x)=\xi\left(\alpha\left(\gamma^{-1}\right)(x)\right)$ Koo31.

A lot of dynamical properties of the action are read from this unitary representation and its spectral properties. For instance, the action is ergodic if and only if its Koopman representation has no $\Gamma$-invariant unit vector. In examples 2.1 and 2.2 or 2.6, various properties are deduced from the fact that the Koopman representation admits a Hilbert basis which is either made of eigenvectors or permuted by $\Gamma$ (see for instance [Sch80], [KT08]). The classical ergodic theory considers such actions up to conjugacy (notation: $\left.\Gamma_{1} \curvearrowright^{\alpha_{1}} X_{1} \stackrel{\text { Conj }}{\sim} \Gamma_{2} \curvearrowright^{\alpha_{2}} X_{2}\right)$.

We now introduce a weaker notion of equivalence and turn from classical ergodic theory to orbit equivalence theory. Here $\Gamma . x$ denotes the orbit of $x$ under the $\Gamma$-action.

Definition 2.8 (Orbit equivalence) Two actions $\Gamma_{i} \curvearrowright^{\alpha_{i}}\left(X_{i}, \mu_{i}\right)$ (for $i=1,2$ ) are orbit equivalent (OE) (notation: $\left.\Gamma_{1} \curvearrowright^{\alpha_{1}} X_{1} \stackrel{O E}{\sim} \Gamma_{2} \curvearrowright^{\alpha_{2}} X_{2}\right)$ if there is a measured space isomorphism $f: X_{1} \rightarrow X_{2}$ that sends orbits to orbits:

$$
\text { for a.a. } x \in X_{1}: f\left(\Gamma_{1} . x\right)=\Gamma_{2} . f(x) .
$$

In particular, the groups are no longer assumed to be isomorphic. When studying actions up to orbit equivalence, what one is really interested in, is the partition of the space into orbits or equivalently the orbit equivalence relation:

$$
\mathcal{R}_{\alpha}:=\{(x, y) \in X: \exists \gamma \in \Gamma \text { s.t. } \alpha(\gamma)(x)=y\} .
$$

\footnotetext{
${ }^{1}$ for instance $X_{0}=\{0,1\}$ and $\mu_{0}(\{0\})=1-p, \mu_{0}(\{1\})=p$ for some $p \in(0,1)$. The only degenerate situation one wishes to avoid is $X_{0}$ consisting of one single atom.

${ }^{2}$ The constants are fixed vectors for the representation on $L^{2}(X, \mu)$. Its orthocomplement $L_{0}^{2}(X, \mu)=$ $L^{2}(X, \mu) \ominus \mathbb{C} 1$ consists in $\left\{\xi \in L^{2}(X, \mu): \int_{X} \xi(x) d \mu(x)=0\right\}$.

${ }^{3} \mathrm{An}$ isomorphism of measure spaces is defined almost everywhere and respects the measures: $f_{*} \mu_{1}=\mu_{2}$.
} 
This equivalence relation satisfies the following three properties: (1) its classes are (at most) countable, (2) as a subset of $X \times X$, it is measurable, (3) it preserves the measure $\mu$ : this means that every measurable automorphism $\phi: X \rightarrow X$ that is inner $(x$ and $\phi(x)$ belong to the same class for a.a. $x \in X$ ) has to preserve $\mu$.

Axiomatically FM77a, the object of study is an equivalence relation $\mathcal{R}$ on $(X, \mu)$ satisfying the above three conditions: we simply call it a p.m.p. equivalence relation. Two p.m.p. equivalence relations $\mathcal{R}_{1}, \mathcal{R}_{2}$ will be orbit equivalent if there is a measured space isomorphism $f: X_{1} \rightarrow X_{2}$ sending classes to classes.

This abstraction is necessary when one wants to consider, for instance, the restriction $\mathcal{R} \mid A$ of $\mathcal{R}$ to some non-null Borel subset $A \subset X$ : the standard Borel space $A$ is equipped with the normalized probability measure $\mu_{A}(C)=\mu(C) / \mu(A)$ and $(x, y) \in \mathcal{R} \mid A \Leftrightarrow x, y \in$ $A$ and $(x, y) \in \mathcal{R}$.

In fact, this more general context allows for much more algebraic flexibility since the lattice of subrelations of $\mathcal{R}_{\alpha}$ for some $\Gamma$-action $\alpha$ is much richer than that of subgroups of $\Gamma$ (see von Neumann's problem in section 10). Also, $\mathcal{R}_{\alpha}$ is easier to decompose as a "free product or a direct product" than $\Gamma$ itself (see section 7 and AG10]).

By an increasing approximation $\mathcal{R}_{n} \nearrow \mathcal{R}$ of a p.m.p. equivalence relation $\mathcal{R}$ we mean an increasing sequence of standard (p.m.p.) equivalence subrelations with $\cup_{n} \mathcal{R}_{n}=$ $\mathcal{R}$.

An important notion is that of hyperfiniteness: a p.m.p. equivalence relation $\mathcal{R}$ is hyperfinite if it admits an increasing approximation by finite equivalence subrelations $\mathcal{R}_{n}$ (i.e. the classes of the $\mathcal{R}_{n}$ are finite). Obviously all the actions of locally finite groups (i.e. groups all of whose finitely generated subgroups are finite) generate orbit equivalence relations in this class; for instance such groups as $\Gamma=\oplus_{\mathbb{N}} \Lambda_{n}$, where the $\Lambda_{n}$ are finite. This is also the case for all $\mathbb{Z}$-actions. Dye's theorem is among the fundamental theorems in orbit equivalence theory:

Theorem 2.9 (Dye59) All the ergodic hyperfinite p.m.p. equivalence relations are mutually orbit equivalent.

A series of results due in particular to Dye, Connes, Krieger, Vershik... leads to OrnsteinWeiss' theorem (see CFW81 for a more general version):

Theorem 2.10 (《OW80) If $\Gamma$ is amenable then all its p.m.p. actions are hyperfinite.

In particular, when ergodic, these actions are indistinguishable from the orbit equivalence point of view! All the usual ergodic theoretic invariants are lost. This common object will be denoted $\mathcal{R}_{\text {hyp }}$. On the other hand, if $\Gamma$ admits a free p.m.p. hyperfinite action, then $\Gamma$ has to be amenable, thus showing the border of this huge singular area that produces essentially a single object. The non-amenable world is much more complicated and richer. 


\section{The full group}

The full group of $\mathcal{R}$ denoted by $[\mathcal{R}]$ is defined as the group of p.m.p. automorphisms of $(X, \mu)$ whose graph is contained in $\mathcal{R}$ :

$$
[\mathcal{R}]:=\{T \in \operatorname{Aut}(X, \mu):(x, T(x)) \in \mathcal{R} \text { for a.a. } x \in X\} .
$$

It was introduced and studied by Dye [Dye59], and it is clearly an OE-invariant. But conversely, its algebraic structure is rich enough to remember the equivalence relation:

Theorem 3.1 (Dye63]) (Dye's reconstruction theorem) Two ergodic p.m.p. equivalence relations $\mathcal{R}_{1}$ and $\mathcal{R}_{2}$ are $O E$ iff their full groups are algebraically isomorphic; moreover the isomorphism is then implemented by an orbit equivalence.

The full group has very nice properties. The topology given by the bi-invariant metric $d(T, S)=\mu\{x: T(x) \neq S(x)\}$ is Polish. In general, it is not locally compact and, in fact, homeomorphic with the separable Hilbert space $\ell^{2}$ KT10.

Theorem 3.2 ([BG80, Kec10]) The full group is a simple group iff $\mathcal{R}$ is ergodic.

And it satisfies this very remarkable, automatic continuity:

Theorem 3.3 (Kittrell-Tsankov [KT10]) If $\mathcal{R}$ is ergodic, then every group homomorphism $f:[\mathcal{R}] \rightarrow G$ with values in a separable topological group is automatically continuous.

Hyperfiniteness translates into an abstract topological group property:

Theorem 3.4 (Giordano-Pestov [GP07]) Assuming $\mathcal{R}$ ergodic, $\mathcal{R}$ is hyperfinite iff $[\mathcal{R}]$ is extremely amenable.

Recall that a topological group $G$ is extremely amenable if every continuous action of $G$ on a (Hausdorff) compact space has a fixed point. Together with Kittrell-Tsankov's result, this gives that every action of $\left[\mathcal{R}_{\text {hyp }}\right]$ by homeomorphisms on a compact metrizable space has a fixed point.

Closely related to the full group, the automorphism group $\operatorname{Aut}(\mathcal{R}):=\{T \in \operatorname{Aut}(X, \mu)$ : $(x, y) \in \mathcal{R} \Rightarrow(T(x), T(y)) \in \mathcal{R}$ for a.a. $x \in X\} \triangleright[\mathcal{R}]$ and the outer automorphism group (the quotient) $\operatorname{Out}(\mathcal{R})=\operatorname{Aut}(\mathcal{R}) /[\mathcal{R}]$ have attracted much attention for several years; see for instance GG88a, Gef93, Gef96, Fur05, IPP08, Pop06b, Kec10, Kid08d, PV08d, PV08a, Gab08 and references therein and section 11.

\section{Associated von Neumann algebra}

In fact, the original interest for orbit equivalence came from its connection with von Neumann algebras. Murray and von Neumann [MvN36] considered p.m.p. group actions $\Gamma \curvearrowright^{\alpha}(X, \mu)$ as a machine to produce finite von Neumann algebras $M_{\alpha}$, via their groupmeasure-space construction. And Singer [Sin55] was the first to explicitly notice that $M_{\alpha}$ only depends on the OE class of the action. Feldman-Moore FM77b extended the group-measure-space construction to the context of p.m.p. equivalence relations. 
A p.m.p. equivalence relation $\mathcal{R}$ on $(X, \mu)$, considered as a Borel subspace of $X \times X$ is naturally equipped with a (a priori infinite) measure $\nu$. It is defined as follows: for every Borel subset $C \subset \mathcal{R}$,

$$
\nu(C)=\int_{X}\left|\pi_{l}^{-1}(x) \cap C\right| d \mu(x),
$$

where $\pi_{l}: \mathcal{R} \rightarrow X$ is the projection onto the first coordinate, $\pi_{l}^{-1}(x)$ is the fiber above $x \in X$, and $\left|\pi_{l}^{-1}(x) \cap C\right|$ is the (at most countable) cardinal of its intersection with $C$. A similar definition could be made with the projection $\pi_{r}$ on the second coordinate instead, but the fact that $\mathcal{R}$ is p.m.p. ensures that these two definitions would coincide.

The (generalized) group-measure-space von Neumann algebra $L(\mathcal{R})$ associated with $\mathcal{R}$ is generated by two families of operators of the separable Hilbert space $L^{2}(\mathcal{R}, \nu)$ : $\left\{L_{g}: g \in[\mathcal{R}]\right\}$ and $\left\{L_{f}: f \in L^{\infty}(X, \mu)\right\}$, where $L_{g} \xi(x, y)=\xi\left(g^{-1} x, y\right)$ and $L_{f} \xi(x, y)=$ $f(x) \xi(x, y)$ for every $\xi \in L^{2}(\mathcal{R}, \nu)$. It contains $\left\{L_{f}: f \in L^{\infty}(X, \mu)\right\} \simeq L^{\infty}(X, \mu)$ as a Cartan subalgebra (i.e. a maximal abelian subalgebra whose normalizer generates $L(\mathcal{R}))$. With this definition, $L(\mathcal{R})$ is clearly an OE-invariant.

Definition 4.1 (von Neumann equivalence or $\mathbf{W}^{*}$-equivalence) Two p.m.p. equivalence relations $\mathcal{R}_{i}$ on $\left(X_{i}, \mu_{i}\right)$ (for $i=1,2$ ) are von Neumann equivalent or $\boldsymbol{W}^{*}$ equivalent if $L\left(\mathcal{R}_{1}\right) \simeq L\left(\mathcal{R}_{2}\right)$ (notation: $\left.\mathcal{R}_{1} \stackrel{v N}{\sim} \mathcal{R}_{2}\right)$.

There exist non-OE equivalence relations producing isomorphic $L(\mathcal{R})$ ([CJ82], OP08b]). Indeed, the additionnal data needed to recover $\mathcal{R}$ is the embedding $L^{\infty}(X, \mu) \subset L(\mathcal{R})$ of the Cartan subalgebra inside $L(\mathcal{R})$ (up to isomorphisms) [Sin55, FM77b].

\section{$5 \quad$ Strong ergodicity}

Recall that a standard p.m.p. equivalence relation $\mathcal{R}$ is ergodic if every $\mathcal{R}$-invariant Borel set $A \subset X$ satisfies $\mu(A)(\mu(A)-1)=0$. The notion of strong ergodicity was introduced by Schmidt as an OE-invariant.

Definition 5.1 ( [Sch80]) An ergodic p.m.p. countable standard equivalence relation $\mathcal{R}$ is strongly ergodic if every almost invariant sequence of Borel subsets $A_{n} \subset X$ is trivial, i.e. satisfies $\lim _{n \rightarrow \infty} \mu\left(A_{n}\right)\left(1-\mu\left(A_{n}\right)\right)=0$.

There are several equivalent definitions of strong ergodicity, see for instance [JS87. We give yet another one below through approximations.

Proposition 5.2 An ergodic equivalence relation $\mathcal{R}$ is strongly ergodic if and only if every increasing approximation $\mathcal{R}_{n} \nearrow \mathcal{R}$ admits an ergodic restriction $\mathcal{R}_{n} \mid U$ to some non-negligeable Borel set $U$, for big enough $n$.

\footnotetext{
${ }^{4}$ for each $g$ in the full group $[\mathcal{R}], \mu(A \Delta g A)=0$.

${ }^{5}$ i.e. for each $g$ in $[\mathcal{R}], \lim _{n \rightarrow \infty} \mu\left(A_{n} \Delta g A_{n}\right)=0$.
} 
In other words, for big enough $n$ the ergodic decomposition of $\mathcal{R}_{n}$ admits an atom. It is easy to see that whenever a p.m.p. action $\Gamma \curvearrowright(X, \mu)$ is non-strongly ergodic, its Koopman representation $\kappa_{0}$ almost has invariant vectors. The converse does not hold in general [Sch81], [HK05]. However, Chifan-Ioana [CI10] extending an argument of AbertNikolov AN07 proved that this is indeed the case when the commutant of $\Gamma \curvearrowright(X, \mu)$ in $\operatorname{Aut}(X, \mu)$ acts ergodically on $(X, \mu)$. Standard Bernoulli shifts are strongly ergodic iff the group is non-amenable. In particular every non-amenable group admits at least one strongly ergodic action.

Kechris-Tsankov [KT08] characterized the generalized Bernoulli shifts $\Gamma \curvearrowright\left(X_{0}, \mu_{0}\right)^{\mathrm{V}}$ that are strongly ergodic as those for which the action $\Gamma \curvearrowright \mathrm{V}$ is non-amenable (i.e. the representation on $\ell^{2}(\mathrm{~V})$ does not admit any sequence of almost invariant vectors).

The consideration of the Koopman representation $\kappa_{0}$ ensures that for (infinite) groups with Kazhdan property (T) every ergodic p.m.p. action is strongly ergodic. And ConnesWeiss (by using Gaussian random variables) showed that this is a criterion for property (T) CW80.

A graphing $\Phi$ (see section (6) on $X$ naturally defines a "metric" $d_{\Phi}$ on $X$ : the simplicial distance associated with the graph structure in the classes of $\mathcal{R}_{\Phi}$ and $d_{\Phi}=\infty$ between two points in different classes. This is a typical instance of what Gromov calls a $\mathbf{m m}$ space Gro00, i.e. a probability measure space $(X, \mu)$ together with a Borel function $d: X \times X \rightarrow \mathbb{R}^{+} \cup\{\infty\}$ satisfying the standard metric axioms except that one allows $d\left(x, x^{\prime}\right)=\infty$. A $m m$-space $(X, \mu, d)$ is concentrated if $\forall \delta>0$, there is $\infty>r_{\delta}>0$ such that $\mu(A), \mu(B) \geq \delta \Rightarrow d(A, B) \leq r_{\delta}$. For instance, if $\Phi=\left\langle\varphi_{1}: X \rightarrow X\right\rangle$ is given by a single p.m.p. ergodic isomorphism, $\left(X, \mu, d_{\Phi}\right)$ is never concentrated. Gromov observed for finitely generated groups that every p.m.p. ergodic action of $\Gamma$ has (respectively, never has) the concentration property if $\Gamma$ has Kazhdan's property ( $T$ ) (respectively, if $\Gamma$ is amenable). Pichot made the connection with strong ergodicity:

Theorem 5.3 ([י[Pic07a] $)$ Let $\Phi=\left(\varphi_{i}\right)_{i=1, \cdots, p}$ be a graphing made of finitely many partial isomorphisms. The space $\left(X, \mu, d_{\Phi}\right)$ is concentrated iff $\mathcal{R}_{\Phi}$ is strongly ergodic.

See also Pic07b for a characterization of strong ergodicity (as well as of property (T) or amenability) in terms of the spectrum of diffusion operators associated with random walks on the equivalence relation $\mathcal{R}$.

For the standard $\operatorname{SL}(2, \mathbb{Z})$ action on the 2 -torus $\mathbb{R}^{2} / \mathbb{Z}^{2}$, every non-amenable subgroup $\Lambda<\mathrm{SL}(2, \mathbb{Z})$ acts ergodically, and even strongly ergodically. Similarly for the generalized Bernoulli shift $\Gamma \curvearrowright\left(X_{0}, \mu_{0}\right)^{\mathrm{V}}$, where the stabilizers of the action $\Gamma \curvearrowright \mathrm{V}$ are amenable. Inspired by [CI10], define more generally:

Definition 5.4 (Solid ergodicity) A p.m.p. standard equivalence relation $\mathcal{R}$ is called solidly ergodic if for every (standard) subrelation $\mathcal{S}$ there exists a measurable partition $\left\{X_{i}\right\}_{i \geq 0}$ of $X$ in $\mathcal{S}$-invariant subsets such that:

(a) the restriction $\mathcal{S} \mid X_{0}$ is hyperfinite

(b) the restrictions $\mathcal{S} \mid X_{i}$ are strongly ergodic for every $i>0$. 
In particular, an ergodic subrelation of a solidly ergodic relation is either hyperfinite or strongly ergodic. By Zimmer [Zim84, Prop. 9.3.2], every ergodic p.m.p. standard equivalence relation $\mathcal{R}$ contains an ergodic hyperfinite subrelation $\mathcal{S}$ which, being non strongly ergodic, contains an aperiodic subrelation with diffuse ergodic decomposition. Thus the $X_{0}$ part cannot be avoided, even for aperiodic subrelations.

One gets an equivalent definition if one replaces "strongly ergodic" by "ergodic" (see [CI10, Prop. 6] for more equivalent definitions). It may seem quite unlikely that such relations really exist. However, Chifan-Ioana [CI10] observed that the notion of solidity and its relative versions introduced by Ozawa Oza04 (by playing between $\mathrm{C}^{*}$ - and von Neumann algebras) imply solid ergodicity (hence the name). Moreover, they established a general solidity result for Bernoulli shifts.

Theorem 5.5 The following actions are solidly ergodic:

- The standard action $\mathrm{SL}(2, \mathbb{Z}) \curvearrowright \mathbb{R}^{2} / \mathbb{Z}^{2}$ Oza09].

- The generalized Bernoulli action $\Gamma \curvearrowright\left(X_{0}, \mu_{0}\right)^{V}$, when the $\Gamma$-action $\Gamma \curvearrowright V$ has amenable stabilizers CI10.

When the group $\Gamma$ is exact $[$, the above statement for the standard Bernoulli shifts also follows from [Oza06, Th. 4.7].

A positive answer to the following percolation-theoretic question would give another proof of solid ergodicity for the standard Bernoulli shifts:

Question 5.6 Let $\Gamma$ be a countable group with a finite generating set $S$. Let $\pi:\left(X_{0}, \mu_{0}\right)^{\Gamma} \rightarrow$ $[0,1]$ be any measure preserving map (i.e. $\pi_{*}\left(\otimes_{\Gamma} \mu_{0}\right)=$ Leb) and $\Phi_{\pi}$ be the "fiber-graphing" made of the restriction $\varphi_{s}$ of $s \in S$ to the set $\left\{\omega \in\left(X_{0}, \mu_{0}\right)^{\Gamma}: \pi(s . \omega)=\pi(\omega)\right\}$. Is the equivalence relation generated by $\Phi_{\pi}$ finite?

\section{Graphings}

The cost of a p.m.p. equivalence relation $\mathcal{R}$ has been introduced by Levitt [Lev95]. It has been studied intensively in [Gab98, Gab00]]. See also [KM04, Kec10, Fur09] and the popularization paper [Gab10b]. When an equivalence relation is generated by a group action, the relations between the generators of the group introduce redundancy in the generation, and one can decrease this redundancy by using instead partially defined isomorphisms.

A countable family $\Phi=\left(\varphi_{j}: A_{i} \stackrel{\sim}{\rightarrow} B_{j}\right)_{j \in J}$ of measure preserving isomorphisms between Borel subsets $A_{i}, B_{i} \subset X$ is called a graphing. It generates a p.m.p. equivalence relation $\mathcal{R}_{\Phi}$ : the smallest equivalence relation such that $x \sim \varphi_{j}(x)$ for $j \in J$ and $x \in A_{j}$. Moreover, $\Phi$ furnishes a graph structure (hence the name) $\Phi[x]$ on the class of each point $x \in X$ : two points $y$ and $z$ in its class are connected by an edge whenever $z=\varphi_{j}^{ \pm 1}(y)$ for some $j \in J$. If $\mathcal{R}$ is generated by a free action of $\Gamma$ and if $\Phi$ is made of isomorphisms associated with a generating set $S$ of $\Gamma$, then the graphs $\Phi[x]$ are isomorphic with the corresponding Cayley graph of $\Gamma$. When all the graphs $\Phi[x]$ are trees, $\Phi$ is called a treeing.

\footnotetext{
${ }^{6}$ Recall that a discrete group $\Gamma$ is exact iff it acts amenably on some compact topological space.
} 
If it admits a generating treeing, $\mathcal{R}$ is called treeable. See Adams Ada88, Ada90 for the first study of treed equivalence relations.

The cost of $\Phi$ is the number of generators weighted by the measure of their support: $\operatorname{Cost}(\Phi)=\sum_{j \in J} \mu\left(A_{j}\right)=\sum_{j \in J} \mu\left(B_{j}\right)$. The cost of $\mathcal{R}$ is the infimum over the costs of its generating graphings: $\operatorname{Cost}(\mathcal{R})=\inf \left\{\operatorname{Cost}(\Phi): \mathcal{R}=\mathcal{R}_{\Phi}\right\}$. It is by definition an OE-invariant. The cost of $\mathcal{R}$ is $\geq 1$ when the classes are infinite [Lev95]. Together with Ornstein-Weiss' theorem this gives that every p.m.p. free action of an infinite amenable group has cost $=1$. Various commutation properties in a group $\Gamma$ also entail cost $=1$ for all of its free actions. For instance when $\Gamma=G \times H$ is the product of two infinite groups and contains at least one infinite order element or $\Gamma=\operatorname{SL}(n, \mathbb{Z})$, for $n \geq 3$. It is not difficult to see that when a finite cost graphing $\Phi$ realizes the cost of $\mathcal{R}_{\Phi}$ then $\Phi$ is a treeing. The main results in Gab98 claim the converse:

Theorem 6.1 () If $\Phi$ is a treeing then $\operatorname{Cost}\left(\mathcal{R}_{\Phi}\right)=\operatorname{Cost}(\Phi)$. In particular, the free actions of the free group $\mathbf{F}_{n}$ have cost $n$.

In particular, free groups of different ranks cannot have OE free actions. The cost measures the amount of information needed to construct $\mathcal{R}$. It is an analogue of the rank of a countable group $\Gamma$, i.e. the minimal number of generators or in a somewhat pedantic formulation, the infimum of the measures $\delta(S)$ over the generating systems $S$, where $\delta$ denotes the counting measure on the group. Similarly the cost of $\mathcal{R}$ is the infimum of the measures $\nu(C)$ over the Borel subsets $C \subset \mathcal{R}$ which generate $\mathcal{R}$, where $\nu$ is the measure on $\mathcal{R}$ introduced in section 4 , equation (2) (compare Connes' Bourbaki seminar [Con04]).

In Gab00a the notion of free product decomposition $\mathcal{R}=\mathcal{R}_{1} * \mathcal{R}_{2}$ (and more generally free product with amalgamation $\mathcal{R}=\mathcal{R}_{1} *_{\mathcal{R}_{3}} \mathcal{R}_{2}$ ) of an equivalence relation over subrelations is introduced (see also [Ghy95, Pau99]). Of course, when $\mathcal{R}$ is generated by a free action of a group, a decomposition of $\Gamma=\Gamma_{1} *_{\Gamma_{3}} \Gamma_{2}$ induces the analogous decomposition of $\mathcal{R}=\mathcal{R}_{\Gamma_{1}} *_{\mathcal{R}_{\Gamma_{3}}} \mathcal{R}_{\Gamma_{2}}$. The cornerstone in cost theory is the following computation:

Theorem 6.2 ( Gab00a $) \operatorname{Cost}\left(\mathcal{R}_{1} *_{\mathcal{R}_{3}} \mathcal{R}_{2}\right)=\operatorname{Cost}\left(\mathcal{R}_{1}\right)+\operatorname{Cost}\left(\mathcal{R}_{2}\right)-\operatorname{Cost}\left(\mathcal{R}_{3}\right)$, when $\mathcal{R}_{3}$ is hyperfinite (possibly trivial).

These techniques allow for the calculation of the cost of the free actions of several groups: for instance $\mathrm{SL}(2, \mathbb{Z})($ Cost $=1+1 / 12)$, surface groups $\pi_{1}\left(\Sigma_{g}\right)($ Cost $=2 g-1) \ldots$ In all the examples computed so far, the cost does not depend on the particular free action of the group, thus raising the following question (which proved to be related to rank gradient and a low-dimensional topology problem; see [AN07]) (see also Question 8.2):

Question 6.3 (Fixed Price Problem) Does there exist a group $\Gamma$ with two p.m.p. free actions of non equal costs?

Observe that both the infimum $\operatorname{Cost}(\Gamma)(\overline{\text { Gab00a }})$ and the supremum $\operatorname{Cost}^{*}(\Gamma)(\overline{\mathrm{AW}})$ among the costs of all free p.m.p. actions of $\Gamma$ are realized by some actions.

Question 6.4 (Cost for Kazdhan groups) Does there exist a Kazdhan property (T) group with a p.m.p. free action of cost $>1$ ? 
In his very rich monograph [Kec10, Kechris studied the continuity properties of the cost function on the space of actions and proved that $\operatorname{Cost}(\mathcal{R})>1$ for an ergodic $\mathcal{R}$ forces its outer automorphism group to be Polish. He also introduced the topological OE-invariant $t([\mathcal{R}])$, defined as the minimum number of generators of a dense subgroup of the full group $[\mathcal{R}]$ and related it with the cost [Kec10]. When $\mathcal{R}$ is generated by a free ergodic action of $\mathbf{F}_{n}$, Miller obtained the following lower bound: $n+1 \leq t([\mathcal{R}])$, and [KT10] proved that $t\left(\left[\mathcal{R}_{\text {hyp }}\right]\right) \leq 3$ and that $t([\mathcal{R}]) \leq 3(n+1)$.

Lyons-Pichot-Vassout [LPV08] introduced the uniform isoperimetric constant $h(\mathcal{R})$ for p.m.p. equivalence relations, a notion similar to that for countable groups $h(\Gamma)$. They were able to obtain the purely group theoretic sharp comparison $2 \beta_{1}^{(2)}(\Gamma) \leq h(\Gamma)$ (where $\beta_{1}^{(2)}(\Gamma)$ is the first $\ell^{2}$-Betti number). Two complementary inequalities from [LPV08, PV09a lead to " $2(\operatorname{Cost}(\mathcal{R})-1)=h(\mathcal{R})$ ", thus identifying two OE-invariants of apparently different nature. See LP09 for an application of cost to percolation theory.

\section{Dimensions}

Geometric group theory studies countable groups through their actions on "nice spaces". Similarly, for a p.m.p. equivalence relation (it is a groupoid [ADR00]) $\mathcal{R}$ on $(X, \mu)$, one might consider its actions on fields of spaces $X \ni x \mapsto \Sigma_{x}$, or $\mathcal{R}$-field. For instance, a graphing $\Phi$ defines a measurable field of graphs $x \mapsto \Phi[x]$, on which the natural isomorphism $\Phi[y] \simeq \Phi[z]$ for $(y, z) \in \mathcal{R}_{\Phi}$ induces an action of $\mathcal{R}_{\Phi}$. The Bass-Serre theory Bas76, Ser77 relates the actions of a group on trees to its free product with amalgamation decompositions (and HNN-extensions). Alvarez Alv09b, Alv09a developped an analogous theory in the framework of equivalence relations. For instance an equivalence relation $\mathcal{R}$ acts "properly" on a field of trees iff $\mathcal{R}$ is treeable Alv09b]. He also obtained a theorem describing the structure of subrelations of a free product Alv09a, analogous to Kurosh's theorem. This led in AG10 to the essential uniqueness of a free product decomposition $\mathcal{R}=\mathcal{R}_{1} * \cdots * \mathcal{R}_{n}$ when the factors are freely indecomposable (i.e. indecomposable as a non-trivial free product) (compare [IPP08, CH10]). See also Sak09b for similar results for some free products with amalgamation over amenable groups.

Definition 7.1 (《G10]) A countable group is called measurably freely indecomposable $(\mathcal{M F I})$ if all its free p.m.p. actions are freely indecomposable.

Examples of $\mathcal{M F} \mathcal{I}$ groups are provided by non-amenable groups with $\beta_{1}^{(2)}=0$.

Question 7.2 ([AG10]) Produce a $\mathcal{M F I}$ group with $\beta_{1}^{(2)}>0$.

More generally, a simplicial $\mathcal{R}$-field is a measurable field of simplicial complexes with a simplicial action of $\mathcal{R}$ (see Gab02): the space $\Sigma^{(0)}$ of 0 -cells has a Borel structure and a measurable map $\pi$ onto $X$ with countable fibers. The cells are defined in the fibers; $\mathcal{R}$ permutes the fibers; and everything is measurable. The action is discrete (or smooth, or proper) if it admits a measurable fundamental domain in $\Sigma^{(0)}$. For example, consider a free p.m.p. action $\Gamma \curvearrowright^{\alpha}(X, \mu)$ and a free action of $\Gamma$ on a (usual, countable) simplicial complex $L$. This defines a proper simplicial $\mathcal{R}_{\alpha}$-action on $X \times L$ induced by the diagonal 
$\Gamma$-action. It is instructive to consider an OE action $\Lambda \curvearrowright^{\beta}(X, \mu)$ and to try to figure out the action of $\mathcal{R}_{\beta}=\mathcal{R}_{\alpha}$ on $X \times L$ once $\Gamma$ is forgotten.

The geometric dimension geo- $\operatorname{dim}(\mathcal{R})$ of $\mathcal{R}$ is defined as the smallest possible dimension of a proper $\mathcal{R}$-field of contractible simplicial complexes [Gab02]. It is analogous to (and bounded above by) the classical geometric dimension ( Bro82]) of $\Gamma$. The approximate dimension [Gab02 (no classical analogue) approx- $\operatorname{dim}(\mathcal{R})$ of $\mathcal{R}$ is defined as the smallest possible upper limit of geometric dimensions along increasing approximations of $\mathcal{R}$ :

$$
\operatorname{approx}-\operatorname{dim}(\mathcal{R}):=\min \left\{\sup \left(\operatorname{geo}-\operatorname{dim}\left(\mathcal{R}_{n}\right)\right)_{n}:\left(\mathcal{R}_{n}\right) \nearrow \mathcal{R}\right\}
$$

For instance, $\operatorname{geo}-\operatorname{dim}(\mathcal{R})=0$ for finite equivalence relations; $\operatorname{approx}-\operatorname{dim}(\mathcal{R})=0$ iff $\mathcal{R}$ is hyperfinite; and geo-dim $(\mathcal{R})=1 \mathrm{iff} \mathcal{R}$ is treeable. Thus, quite surprisingly, surface groups admit free actions of geo-dim $=1$. Every free action of a Kazhdan property $(\mathrm{T})$ group satisfies approx-dim $=$ geo-dim $>1$ AS90, Moo82, Gab10a. In the following statement, $\beta_{n}^{(2)}$ denotes the $n$-th $\ell^{2}$-Betti number (see section 8 ).

Theorem 7.3 ( Gab10a) These dimensions satisfy:

$-a-\operatorname{geo-} \operatorname{dim}(\mathcal{R})-1 \leq \operatorname{approx}-\operatorname{dim}(\mathcal{R}) \leq \operatorname{geo}-\operatorname{dim}(\mathcal{R})$.

-b- If $\Lambda<\Gamma$ satisfies $\beta_{p}^{(2)}(\Lambda) \neq 0$, then geo-dim $\left(\mathcal{R}_{\alpha}\right) \geq p$ for every free $p . m . p$. action $\Gamma \curvearrowright^{\alpha}(X, \mu)$. If moreover geo- $\operatorname{dim}\left(\mathcal{R}_{\alpha}\right)=p$, then $\beta_{p}^{(2)}(\Gamma) \neq 0$.

It follows that every free action action of $\mathbf{F}_{r_{1}} \times \cdots \times \mathbf{F}_{r_{p}}\left(r_{j} \geq 2\right)$ (resp. $\mathbb{Z} \times \mathbf{F}_{r_{1}} \times \cdots \times \mathbf{F}_{r_{p}}$ ) has approx-dim $=$ geo-dim $=p$ (resp. geo-dim $=p+1$ ). Moreover, for every $p \geq 3$, there is a group $\Gamma_{p}$ with free actions $\alpha_{p}$ and $\beta_{p}$ such that $\operatorname{approx}-\operatorname{dim}\left(\mathcal{R}_{\alpha_{p}}\right)=\operatorname{geo}-\operatorname{dim}\left(\mathcal{R}_{\alpha_{p}}\right)=p$ and $\operatorname{approx}-\operatorname{dim}\left(\mathcal{R}_{\beta_{p}}\right)+1=\operatorname{geo}-\operatorname{dim}\left(\mathcal{R}_{\beta_{p}}\right)=p$.

In [DG09], Dooley-Golodets study the behavior of the dimension geo-dim under finite extensions. The notion of measurable cohomological dimension introduced in ST07] has some similarity with the geometric dimension.

\section{$8 \quad L^{2}$-Betti numbers}

The $\ell^{2}$-Betti numbers of cocompact group actions on manifolds were introduced by Atiyah Ati76] in terms of the heat kernel. Connes [Con79] defined them for measured foliations. Cheeger-Gromov CG86 introduced $\ell^{2}$-Betti numbers $\beta_{n}^{(2)}(\Gamma) \in[0, \infty], n \in \mathbb{N}$, for arbitrary countable groups $\Gamma$. In Gab02 the $L^{2}$-Betti numbers $\beta_{n}^{(2)}(\mathcal{R}) \in[0, \infty], n \in \mathbb{N}$ are defined for p.m.p. equivalence relations $\mathcal{R}$, by using proper simplicial $\mathcal{R}$-fields (see section $\mathbb{0}$ ). In any case, the definitions rely on the notion of generalized von Neumann dimension, expressed as the trace of certain projections. One of the main results in Gab00b, Gab02 is the invariance of the $\beta_{n}^{(2)}(\Gamma)$ under orbit equivalence.

Theorem 8.1 ( Gab02] ) If $\mathcal{R}_{\Gamma}$ is generated by a free p.m.p. action of $\Gamma$, then $\beta_{n}^{(2)}\left(\mathcal{R}_{\Gamma}\right)=$ $\beta_{n}^{(2)}(\Gamma)$ for every $n \in \mathbb{N}$.

The inequality $\operatorname{Cost}(\Gamma) \geq \beta_{1}^{(2)}(\Gamma)-\beta_{0}^{(2)}(\Gamma)+1$ proved in Gab02 is an equality in all cases where the computations have been achieved, thus leading to the question: 
Question 8.2 (Cost vs first $\ell^{2}$-Betti number) Is there an infinite countable group with $\operatorname{Cost}(\Gamma)>\beta_{1}^{(2)}(\Gamma)+1$ ?

The following compression formula was a key point in various places notably when studying "self-similarities" (the "fundamental group", see [Pop06a]) and measure equivalence (see section 9).

Theorem 8.3 ([Gab02]) The $L^{2}$-Betti numbers of $\mathcal{R}$ and of its restriction to a Borel subset $A \subset X$ meeting all the classes satisfy: $\beta_{n}^{(2)}(\mathcal{R})=\mu(A) \beta_{n}^{(2)}(\mathcal{R} \mid A)$.

It follows that lattices in a common locally compact second countable group have proportional $\ell^{2}$-Betti numbers.

In BG04, $L^{2}$-Betti numbers for profinite actions are used to extend Lück's approximation theorem [ü̈c94 to non-normal subgroups. We refer to the book [Lüc02 for information about $\ell^{2}$-Betti numbers of groups and for an alternative approach to von Neumann dimension. See [Sau05, ST07, Tho08] for extension of $\beta_{n}^{(2)}(\mathcal{R})$ to measured groupoids, and several computations using Lück's approach ( NR09 proves that the various definitions coincide).

Very interesting combinatorial analogues of the cost and $\beta_{1}^{(2)}$ have been introduced by Elek [Ele07] in a context of sequences of finite graphs.

\section{Measure equivalence}

Two groups $\Gamma_{1}$ and $\Gamma_{2}$ are virtually isomorphic if there exist $F_{i} \triangleleft \Lambda_{i}<\Gamma_{i}$ such that $\Lambda_{1} / F_{1} \simeq \Lambda_{2} / F_{2}$, where $F_{i}$ are finite groups, and $\Lambda_{i}$ has finite index in $\Gamma_{i}$. This condition is equivalent with: $\Gamma, \Lambda$ admit commuting actions on a set $\Omega$ such that each of the actions $\Gamma \curvearrowright \Omega$ and $\Lambda \curvearrowright \Omega$ has finite quotient set and finite stabilizers.

A finite set admits two natural generalizations, a topological one (compact set) leading to geometric group theory and a measure theoretic one (finite measure set) leading to measured group theory.

Definition 9.1 (Gro93) Two countable groups $\Gamma_{1}$ and $\Gamma_{2}$ are measure equivalent (ME) (notation: $\Gamma_{1} \stackrel{M E}{\sim} \Gamma_{2}$ ) if there exist commuting actions of $\Gamma_{1}$ and $\Gamma_{2}$, that are (each) measure preserving, free, and with a finite measure fundamental domain, on some standard (infinite) measure space $(\Omega, m)$.

The ratio $\left[\Gamma_{1}: \Gamma_{2}\right]_{\Omega}:=m\left(\Omega / \Gamma_{2}\right) / m\left(\Omega / \Gamma_{1}\right)$ of the measures of the fundamental domains is called the index of the coupling $\Omega$. The typical examples, besides virtually isomorphic groups, are lattices in a common (locally compact second countable) group $G$ with its Haar measure, acting by left and right multiplication.

The topological analogue was shown to be equivalent with quasi-isometry (QI) between finitely generated groups Gro93, thus raising measured group theory (i.e. the study of groups up to ME) to parallel geometric group theory. See Fur99a for the basis in ME and the surveys Gab05a, Sha05, Fur09 for more recent developments. Measure equivalence and orbit equivalence are intimately connected by considering the 
relation between the quotient actions $\Gamma_{1} \curvearrowright \Omega / \Gamma_{2}$ and $\Gamma_{2} \curvearrowright \Omega / \Gamma_{1}$. In fact two groups are ME iff they admit SOE free actions.

Definition 9.2 (Stable Orbit Equivalence) Two p.m.p. actions of $\Gamma_{i} \curvearrowright\left(X_{i}, \mu_{i}\right)$ are stably orbit equivalent (SOE) if there are Borel subsets $Y_{i} \subset X_{i}, i=1,2$ which meet almost every orbit of $\Gamma_{i}$ and a measure-scaling isomorphism $f: Y_{1} \rightarrow Y_{2}$ s.t.

$$
f\left(\Gamma_{1} . x \cap Y_{1}\right)=\Gamma_{2} . f(x) \cap Y_{2} \quad \text { a.e. }
$$

The index or compression constant of this SOE $f$ is $\left[\Gamma_{1}: \Gamma_{2}\right]_{f}=\frac{\mu\left(Y_{2}\right)}{\mu\left(Y_{1}\right)}$.

The state of the art ranges from quite well understood ME-classes to mysterious and very rich examples. For instance, the finite groups obviously form a single ME-class. The infinite amenable groups form a single ME-class OW80. The ME-class of a lattice in a center-free simple Lie group $G$ with real rank $\geq 2$ (like $\operatorname{SL}(n, \mathbb{R}), n \geq 3)$ consists in those groups that are virtually isomorphic with a lattice in $G$ Fur99a. If $\Gamma$ is a non-exceptional mapping class group, its ME-class consists only in its virtual isomorphism class [Kid08a]. Kida extended this kind of result to some amalgamated free products (see Kid09).

On the opposite, the ME-class of the (mutually virtually isomorphic) free groups $\mathbf{F}_{r}$ $(2 \leq r<\infty)$ contains the free products $*_{i=1}^{r} A_{i}$ of infinite amenable groups, surface groups $\pi_{1}\left(\Sigma_{g}\right)(g \geq 2)$, certain branched surface groups Gab05a, elementarily free groups BTW07... and is far from being understood. Being ME with a free group is equivalent to admitting a free p.m.p. treeable action Hjo06].

There is a considerable list of ME-invariants (see Gab05a and the references therein). For instance Kazhdan property (T), Haagerup property, the ergodic dimension (resp. approximate ergodic dimension) defined as the infimum of the geometric (resp. approximate) dimension among all the free p.m.p. actions of $\Gamma$, the sign of the Euler characteristic (when defined), the Cowling-Haagerup invariant, belonging to the classes $\mathcal{C}_{\text {reg }}, \mathcal{C}$. Recently exactness (see [BO08]) and belonging to the class $\mathcal{S}$ of Ozawa Sak09a were proved to be ME-invariants. There are also numerical invariants which are preserved under ME modulo multiplication by the index: $\operatorname{Cost}(\Gamma)-1$, the $\ell^{2}$-Betti numbers $\left(\beta_{n}^{(2)}(\Gamma)\right)_{n \in \mathbb{N}}$ Gab02.

ME is stable under some basic constructions:

(a) if $\Gamma_{i} \stackrel{\text { ME }}{\sim} \Lambda_{i}$ for $i=1, \cdots, n$ then $\Gamma_{1} \times \cdots \times \Gamma_{n} \stackrel{\text { ME }}{\sim} \Lambda_{1} \times \cdots \times \Lambda_{n}$

(b) if $\Gamma_{i} \stackrel{\mathrm{ME}}{\sim} \Lambda_{i}$ with index 1 , then $\Gamma_{1} * \cdots * \Gamma_{n} \stackrel{\mathrm{ME}}{\sim} \Lambda_{1} * \cdots * \Lambda_{n}$ (with index 1).

Some papers study when the converse holds [MS06, [PP08, CH10, AG10]. One has of course to impose some irreducibility conditions on the building blocks, and these conditions have to be strong enough to resist the measurable treatment. These requirements are achieved

(a) (for direct products) if the $\Gamma_{i}, \Lambda_{i}$ belong to the class $\mathcal{C}_{\text {reg }}$ of [MS06] (for instance if they are non-amenable non-trivial free products): the non-triviality of the bounded cohomology $\mathrm{H}_{\mathrm{b}}^{2}\left(\Gamma, \ell^{2}(\Gamma)\right)$ is an ME-invariant preventing $\Gamma$ to decompose (non-trivially) as a direct product;

(b) (for free products) if the $\Gamma_{i}, \Lambda_{i}$ are $\mathcal{M F I}$ (for instance if they have $\beta_{1}^{(2)}=0$ and are non-amenable) [AG10]: they are not ME with a (non-trivial) free product. We prove for instance: 
Theorem 9.3 ([AG10]) If $\Gamma_{1} * \cdots * \Gamma_{n} \stackrel{M E}{\sim} \Lambda_{1} * \cdots * \Lambda_{p}$, where both the $\Gamma_{i}$ 's and the $\Lambda_{j}$ 's belong to distinct $M E$-classes and are $\mathcal{M F I}$, then $n=p$ and up to a permutation of the indices $\Gamma_{i} \stackrel{M E}{\sim} \Lambda_{i}$.

See also [LP08, CH10] when the groups have Kazhdan property (T), or are direct products, under extra ergodicity hypothesis. The delicate point of removing ergodicity assumptions in [AG10] was achieved by using [Alv09a.

Similar "deconstruction" results were obtained by Sako Sak09b for building blocks made of direct products of non-amenable exact groups when considering free products with amalgamation over amenable subgroups or by taking wreath product with amenable base.

Refinements of the notion of ME were introduced in [Sha04, Tho09, LSW09] or by Sauer and Bader-Furman-Sauer.

\section{Non-orbit equivalent actions for a given group}

In this section, we only consider ergodic free p.m.p. actions $\Gamma \curvearrowright^{\alpha}(X, \mu)$ of infinite countable groups and the associated orbit equivalence relations $\mathcal{R}_{\alpha}$. Ornstein-Weiss' theorem OW80 implies that amenable groups all produce the same relation, namely $\mathcal{R}_{\text {hyp }}$. What about non-amenable groups? How many non-OE actions for a given group? Most of the OEinvariants depend on the group rather than on the action, and thus cannot distinguish between various actions of the group. However, for non-Kazhdan property $(\mathrm{T})$ groups, Connes-Weiss CW80 produced two non-OE actions distinguished by strong ergodicity (see section [). And along the years, various rigidity results entailed some specific families of groups to admit continously] many non-OE actions (see for instance [BG81, Zim84, GG88b, MS06, Pop06b, Pop07a ).

We briefly describe below the crucial steps on the route toward the general solution:

Theorem 10.1 ([Гוoa07, Eps08]) Every non-amenable group admits continuously many orbit inequivalent free ergodic p.m.p. actions.

The first step was made by Hjorth [Hjo05] when, within the circle of ideas from Connes [Con80] and Popa [Pop86], he obtained the result for Kazhdan property (T) groups. Roughly speaking, a pair of OE actions $\alpha$ and $\beta$ defining the same equivalence relation $\mathcal{R}$ gives a diagonal action $\left(\gamma \cdot(x, y)=\left(\gamma \cdot{ }_{\alpha} x, \gamma \cdot{ }_{\beta} y\right)\right)$ on $\mathcal{R}$ and thus a unitary representation on $L^{2}(\mathcal{R}, \nu)$. When considering uncountably many OE actions, a separability argument shows that the characteristic function $\mathbf{1}_{D}$ of the diagonal is sufficiently almost invariant for some pair of actions. Now, an invariant vector near $\mathbf{1}_{D}$, which is given by property $(\mathrm{T})$, delivers a conjugacy between the actions. There exists a continuum of pairwise non-conjugate actions, and by the above the OE-classes in this continuum are countable.

The next step was the analogous theorem for the prototypical non-property $(\mathrm{T})$, nonrigid group, namely the free groups and some free products [GP05]. It lay again within

\footnotetext{
${ }^{7}$ This is an upper bound since $\operatorname{Card}(\operatorname{Aut}([0,1]$, Leb $))=2^{\aleph_{0}}$.
} 
the same circle of ideas but there, rigidity was obtained through Popa's property $(\mathbf{T})$ relative to the space (see section 11).

Then Ioana [oa07] extended it to all groups containing a copy of $\mathbf{F}_{2}$. For this, he introduced a weak version of property $(\mathrm{T})$ relative to the space and used a general construction called co-induction?.

Eventually, Epstein obtained the theorem in full generality Eps08. For this she had to generalize the co-induction construction to the setting provided by Gaboriau-Lyons' measurable solution to von Neumann's problem (see below). Moreover, Ioana extended Epstein's result from orbit inequivalent to von Neumann inequivalent actions [oa07.

When von Neumann introduced the notion of amenability vN29, he observed that a countable group containing a copy of $\mathbf{F}_{2}$ cannot be amenable. The question of knowing whether every non-amenable countable group has to contain a copy of $\mathbf{F}_{2}$, known as von Neumann's problem, was answered in the negative by $\mathrm{Ol}^{\prime}$ šanskiı $\mathrm{Ol}^{\prime} 80$. In the measurable framework, offering much more flexibility, the answer is somewhat different:

Theorem 10.2 (GL09]) For any non-amenable countable group $\Gamma$, the orbit equivalence relation of the Bernoulli shift action $\Gamma \curvearrowright([0,1], \text { Leb })^{\Gamma}$ contains a subrelation generated by a free ergodic p.m.p. action of $\mathbf{F}_{2}$.

In the terminology of Mon06, there is a randembedding of $\mathbf{F}_{2}$ in any non-amenable group. The proof uses percolation theory on graphs and HP99, LS99, PSN00, Gab05b, Hjo06]. The following general question remains open:

Question 10.3 Does every ergodic non-hyperfinite p.m.p. equivalence relation contain a (treeable) subrelation of cost $>1$ ?

\section{Relative property (T)}

In his seminal paper [Kaz67] on property $(\mathrm{T})$, Kazhdan implicitly 9 introduced the notion of property ( $\mathrm{T}$ ) relative to a subgroup $\Lambda<\Gamma$. In particular, a group always has property (T) relative to its "unit subgroup" $\{1\}<\Gamma$. When considering a groupoid like $\mathcal{R}$, its space of units $(X, \mu)$ (and its "relative representation theory") is much more complicated. The introduction by Popa Pop06a of the fruitful notion of property (T) relative to the space $(X, \mu)$ (also simply called rigidity) allowed him to solve some long standing problems in von Neumann algebras. In fact, the definition involves a pair of von Neumann algebras $B \subset M$ (for instance $L^{\infty}(X, \mu) \subset L(\mathcal{R})$ ) and parallels the analogous notion for groups, in the spirit of Connes-Jones CJ85].

The typical example is provided by the standard action of $\mathrm{SL}(2, \mathbb{Z})$ and its nonamenable subgroups $\Gamma$ (for instance free groups $\mathbf{F}_{r}, r \geq 2$ ) on $\mathbb{T}^{2}$. Notice Ioana's result that in fact every ergodic non-amenable subrelation of $\mathcal{R}_{\mathrm{SL}(2, \mathbb{Z}) \curvearrowright \mathbb{T}^{2}}$ still has property $(\mathrm{T})$ relative to the space $\mathbb{T}^{2}$ [oa09]. The property $(\mathrm{T})$ relative to the space $(X, \mu)$ comes

\footnotetext{
${ }^{8} \mathrm{Co}$-induction is the classical right adjoint of restriction. Its measure theoretic version was brought to my attention by Sauer and used in Gab05a, but it probably first appeared in preliminary versions of DGRS08.

${ }^{9}$ This was made explicit in Mar82].
} 
from the group property (T) of $\mathbb{Z}^{2} \rtimes \Gamma$ relative to the subgroup $\mathbb{Z}^{2}$, via viewing $\mathbb{Z}^{2}$ as the Pontryagin dual of $\mathbb{T}^{2}$. This property (never satisfied by standard Bernoulli shifts) entails several rigidity phenomena (see for instance [Pop06a, [PP08, GP05]). More examples come from Val05, Fer06 and they all involve some arithmeticity. This led Popa to ask for the class of groups admitting such a free p.m.p. action with property (T) relative to the space. Törnquist Tör06 ensures that the class is stable under taking a free product with any countable group. More generally, Gab08 shows that the class contains all the non-trivial free products of groups $\Gamma=\Gamma_{1} * \Gamma_{2}$ : in fact $\mathcal{R}_{\Gamma_{1}}$ and $\mathcal{R}_{\Gamma_{2}}$ may be chosen to be conjugate with any prescribed free $\Gamma_{i}$-action and the arithmeticity alluded to is hidden in the way they are put in free product. This leads, using ideas from [PV08a to (plenty of) examples of $\mathcal{R}_{\Gamma}$ with trivial outer automorphism group, in particular the first examples for free $\mathbf{F}_{2}$-actions [Gab08. Ioana [Ioa07] proved that every non-amenable group admits a free p.m.p. action satisfying a weak form of the above property, enough for various purposes, see section 10 .

\section{Some rigidity results}

We have three notions of equivalence between free p.m.p. actions:

$$
\left(\Gamma_{1} \curvearrowright^{\alpha_{1}} X_{1} \stackrel{\text { Conj }}{\sim} \Gamma_{2} \curvearrowright^{\alpha_{2}} X_{2}\right) \Longrightarrow\left(\Gamma_{1} \curvearrowright^{\alpha_{1}} X_{1} \stackrel{\mathrm{OE}}{\sim} \Gamma_{2} \curvearrowright^{\alpha_{2}} X_{2}\right) \Longrightarrow\left(\mathcal{R}_{\alpha_{1}} \stackrel{\mathrm{vN}}{\sim} \mathcal{R}_{\alpha_{2}}\right) .
$$

Rigidity phenomena consist ideally in situations where (for free actions) some implication can be reversed, or more generally when a big piece of information of a stronger nature can be transferred through a weaker equivalence. Zimmer's pioneering work (see [Zim84]) inaugurated a series of impressive results of rigidity for the first arrow $(\stackrel{\text { Conj }}{\sim} \leftarrow-\stackrel{\text { OE }}{\sim})$, made possible by the introduction in OE theory and in operator algebras of new techniques borrowed from diverse mathematical domains, like algebraic groups, geometry, geometric group theory, representation theory or operator algebras. These rigidity results for $\Gamma_{1} \curvearrowright^{\alpha_{1}} X_{1}$ take various qualifications according to whether an OE hypothesis entails - strong OE rigidity: conjugacy under some additionnal hypothesis about the mysterious action $\Gamma_{2} \curvearrowright^{\alpha_{2}} X_{2}$, or even

- OE superrigidity: conjugacy of the actions with no hypothesis at all on the target action.

These notions are virtual when they happen only up to finite groups (see Fur99b for precise definitions).

To give some ideas we simply evoke a sample of some typical and strong statements far from exhaustiveness or full generality.

Theorem 12.1 ( Fur99b) Any free action that is $O E$ with the standard action $\mathrm{SL}(n, \mathbb{Z}) \curvearrowright$ $\mathbb{T}^{n}$ for $n \geq 3$, is virtually conjugate with it.

This is more generally true for lattices in a connected, center-free, simple, Lie group of higher rank, and for "generic" actions (see [Fur99b). Monod-Shalom MS06] obtained strong OE rigidity results when $\Gamma_{1}$ is a direct product of groups in $\mathcal{C}_{\text {reg }}$, under 
appropriate ergodicity assumptions on both sides. See also Hjorth-Kechris HK05 for rigidity results about actions of products, where the focus is more on Borel reducibility. Kida's results Kid08b consider actions of mapping class groups of orientable surfaces and their direct products. He also obtains very strong rigidity results for certain amalgamated free products [Kid09]. A series of ground breaking results in von Neumann algebras obtained by Popa [Pop06a, Pop06a, Pop06d, Pop07a, Pop08] and his collaborators [PS07, IPP08, PV08d, PV08b, PV08a, Ioa08, PV08d, PV09b (see Vae07 for a review) dramatically modified the landscape. On the OE side, these culminated in Popa's cocycle superrigidity theorems, that imply several impressive OE superrigidity corollaries, for instance:

Theorem 12.2 ( Pop07a, Pop08) Assume that $\Gamma$ is either an infinite ICC Kazhdan property (T) group or is the product of two infinite groups $H \times H^{\prime}$ and has no finite normal subgroup. Then any free action that is orbit equivalent with the Bernoulli shift $\Gamma \curvearrowright\left(X_{0}, \mu_{0}\right)^{\Gamma}$ is conjugate with it.

See Furman's ergodic theoretical treatment and generalizations [Fur07 for the Kazhdan property ( $\mathrm{T}$ ) case. In the opposite direction, Bowen obtained some surprising non-rigidity results Bow09a, Bow09b showing for instance that all the Bernoulli shifts of the free groups $\mathbf{F}_{r}, 2 \leq r<\infty$ are mutually SOE (see Def. 9.2).

As it follows from Sin55, FM77b, being able to reverse the second arrow $(\stackrel{\mathrm{OE}}{\sim} \leftarrow--\stackrel{\mathrm{vN}}{\sim})$ essentially amounts to being able to uniquely identify the Cartan subalgebra inside $L(\mathcal{R})$, i.e. given two Cartan subalgebras $A_{1}, A_{2}$ in $L\left(\mathcal{R}_{1}\right) \simeq L\left(\mathcal{R}_{2}\right)$, being able to relate them through the isomorphism. Such results are qualified vNE rigidity or $W^{*}$-rigidity. The starting point is Popa's breakthrough Pop06a where a uniqueness result is obtained under some hypothesis on both $A_{1}$ and $A_{2}$ (and this was enough to solve long standing problems in von Neumann algebras). See also [IPP08, CH10] for this kind of strong statements under various quite general conditions. We refer to the surveys Pop07b, Vae07, Vae10 for the recent developments in $\mathrm{vNE}$ or $W^{*}$-rigidity. However, after a series of progresses (see for instance [OP08a, OP08b, [oa08, Pet09, PV09b, Pet10]), the most recent achievement is:

Theorem 12.3 ([וoa10]) If a free action of a group is von Neumann equivalent with the standard Bernoulli shift action of an ICC Kazhdan property (T) group, then the actions are in fact conjugate.

\section{Some further OE-invariants}

In order to distinguish treeable Borel equivalence relations, Hjorth introduced a technique preventing a p.m.p. equivalence relation from being $\mathrm{OE}$ with a profinite one Hjo06]. Then Kechris and Epstein-Tsankov isolated representation-theoretic properties (i.e. in terms of the Koopman representation) leading to strong forms of non-profiniteness; see Kec05, ET10.

Elek-Lippner introduced the sofic property for equivalence relations. It is satisfied by profinite actions, treeable equivalence relations and Bernoulli shifts of sofic groups 
EL10]. They also proved that the associated von Neumann algebra satisfies the Connes' embedding conjecture.

Acknowledgements I'm grateful to A. Alvarez, C. Houdayer and J. Melleray for their comments.

\section{References}

[Ada88] S. Adams. Indecomposability of treed equivalence relations. Israel J. Math., 64(3):362-380 (1989), 1988.

[Ada90] S. Adams. Trees and amenable equivalence relations. Ergodic Theory Dynamical Systems, 10(1):1-14, 1990.

[ADR00] C. Anantharaman-Delaroche and J. Renault. Amenable groupoids, volume 36 of Monographies de L'Enseignement Mathématique. L'Enseignement Mathématique, Geneva, 2000.

[AG10] A. Alvarez and D. Gaboriau. Free products, orbit equivalence and measure equivalence rigidity. to appear in G.G.D., 2010.

[Alv09a] A. Alvarez. Un théorème de Kurosh pour les relations d'équivalence boréliennes. to appear in Annales de l'Institut Fourier, 2009.

[Alv09b] A. Alvarez. Une théorie de Bass-Serre pour les groupoïdes boréliens. preprint, 2009 .

[AN07] M. Abert and N. Nikolov. Rank gradient, cost of groups and the rank versus Heegaard genus problem. preprint, 2007.

[AS90] S. Adams and R. Spatzier. Kazhdan groups, cocycles and trees. Amer. J. Math., 112(2):271-287, 1990.

[Ati76] M. Atiyah. Elliptic operators, discrete groups and von Neumann algebras. In Colloque "Analyse et Topologie" en l'Honneur de Henri Cartan (Orsay, 1974), pages 43-72. Astérisque, SMF, No. 32-33. Soc. Math. France, Paris, 1976.

[AW] M. Abert and B. Weiss. Bernoulli actions are weakly contained in any free action. in preparation.

[Bas76] H. Bass. Some remarks on group actions on trees. Comm. Algebra, 4(12):10911126, 1976.

[BG80] S. I. Bezuglyı̆ and V. Ja. Golodec. Topological properties of complete groups of automorphisms of a space with a measure. Siberian Math. J., 21(2):147-155, 1980 .

[BG81] S. I. Bezuglyı̆ and V. Ya. Golodets. Hyperfinite and $\mathrm{II}_{1}$ actions for nonamenable groups. J. Funct. Anal., 40(1):30-44, 1981. 
[BG04] N. Bergeron and D. Gaboriau. Asymptotique des nombres de Betti, invariants $l^{2}$ et laminations. Comment. Math. Helv., 79(2):362-395, 2004.

[BO08] N. P. Brown and N. Ozawa. $C^{*}$-algebras and finite-dimensional approximations, volume 88 of Graduate Studies in Mathematics. A.M.S., 2008.

[Bow09a] L. Bowen. Orbit equivalence, coinduced actions and free products. to appear in Groups Geom. Dyn., 2009.

[Bow09b] L. Bowen. Stable orbit equivalence of Bernoulli shifts over free groups. to appear in Groups Geom. Dyn., 2009.

[Bro82] K. Brown. Cohomology of groups. Springer-Verlag, New York, 1982.

[BTW07] M. R. Bridson, M. Tweedale, and H. Wilton. Limit groups, positive-genus towers and measure-equivalence. Ergodic Theory Dynam. Systems, 27(3):703$712,2007$.

[CFW81] A. Connes, J. Feldman, and B. Weiss. An amenable equivalence relation is generated by a single transformation. Ergodic Theory Dynamical Systems, 1(4):431-450 (1982), 1981.

[CG86] J. Cheeger and M. Gromov. $L_{2}$-cohomology and group cohomology. Topology, 25(2):189-215, 1986.

[CH10] I. Chifan and C. Houdayer. Bass-Serre rigidity results in von Neumann algebras. to appear in Duke Math. J., 2010.

[CI10] I. Chifan and A. Ioana. Ergodic subequivalence relations induced by a Bernoulli action. to appear in Geom. and Funct. Anal., 2010.

[CJ82] A. Connes and V. Jones. A $\mathrm{II}_{1}$ factor with two nonconjugate Cartan subalgebras. Bull. Amer. Math. Soc. (N.S.), 6(2):211-212, 1982.

[CJ85] A. Connes and V. Jones. Property $T$ for von Neumann algebras. Bull. London Math. Soc., 17(1):57-62, 1985.

[Con79] A. Connes. Sur la théorie non commutative de l'intégration. In Algèbres d'opérateurs (Sém., Les Plans-sur-Bex, 1978), pages 19-143. Springer, Berlin, 1979 .

[Con80] A. Connes. A factor of type $\mathrm{II}_{1}$ with countable fundamental group. J. Operator Theory, 4(1):151-153, 1980.

[Con04] A. Connes. Nombres de Betti $L^{2}$ et facteurs de type $\mathrm{II}_{1}$ (d'après D. Gaboriau et S. Popa). Number 294, pages ix, 321-333. S.M.F., 2004.

[CW80] A. Connes and B. Weiss. Property T and asymptotically invariant sequences. Israel J. Math., 37(3):209-210, 1980. 
[DG09] A. H. Dooley and V. Ya. Golodets. The geometric dimension of an equivalence relation and finite extensions of countable groups. Ergodic Theory Dynam. Systems, 29(6):1789-1814, 2009.

[DGRS08] A. H. Dooley, V. Ya. Golodets, D. J. Rudolph, and S. D. Sinel'shchikov. NonBernoulli systems with completely positive entropy. Ergodic Theory Dynam. Systems, 28(1):87-124, 2008.

[Dye59] H. Dye. On groups of measure preserving transformation. I. Amer. J. Math., 81:119-159, 1959.

[Dye63] H. Dye. On groups of measure preserving transformations. II. Amer. J. Math., 85:551-576, 1963.

[EL10] G. Elek and G. Lippner. Sofic equivalence relations. J. Funct. Anal., 258(5):1692-1708, 2010.

[Ele07] G. Elek. The combinatorial cost. Enseign. Math. (2), 53(3-4):225-235, 2007.

[EM09] I. Epstein and N. Monod. Nonunitarizable representations and random forests. Int. Math. Res. Not. IMRN, (22):4336-4353, 2009.

[Eps08] I. Epstein. Orbit inequivalent actions of non-amenable groups. preprint, 2008.

[ET10] I. Epstein and T. Tsankov. Modular actions and amenable representations. Trans. Amer. Math. Soc., 362(2):603-621, 2010.

[Fer06] T. Fernós. Relative property (T) and linear groups. Ann. Inst. Fourier (Grenoble), 56(6):1767-1804, 2006.

[FM77a] J. Feldman and C. Moore. Ergodic equivalence relations, cohomology, and von Neumann algebras. I. Trans. Amer. Math. Soc., 234(2):289-324, 1977.

[FM77b] J. Feldman and C. Moore. Ergodic equivalence relations, cohomology, and von Neumann algebras. II. Trans. Amer. Math. Soc., 234(2):325-359, 1977.

[Fur99a] A. Furman. Gromov's measure equivalence and rigidity of higher rank lattices. Ann. of Math. (2), 150(3):1059-1081, 1999.

[Fur99b] A. Furman. Orbit equivalence rigidity. Ann. of Math. (2), 150(3):1083-1108, 1999.

[Fur05] A. Furman. Outer automorphism groups of some ergodic equivalence relations. Comment. Math. Helv., 80(1):157-196, 2005.

[Fur07] A. Furman. On Popa's cocycle superrigidity theorem. Int. Math. Res. Not. IMRN, (19):Art. ID rnm073, 46, 2007.

[Fur09] A. Furman. A survey of measured group theory. Proceedings of a Conference honoring Robert Zimmer's 60th birthday, arXiv:0901.0678v1 [math.DS], 2009. 
[Gab98] D. Gaboriau. Mercuriale de groupes et de relations. C. R. Acad. Sci. Paris Sér. I Math., 326(2):219-222, 1998.

[Gab00a] D. Gaboriau. Coût des relations d'équivalence et des groupes. Invent. Math., 139(1):41-98, 2000.

[Gab00b] D. Gaboriau. Sur la (co-)homologie $L^{2}$ des actions préservant une mesure. $C$. R. Acad. Sci. Paris Sér. I Math., 330(5):365-370, 2000.

[Gab02] D. Gaboriau. Invariants $L^{2}$ de relations d'équivalence et de groupes. Publ. Math. Inst. Hautes Études Sci., 95:93-150, 2002.

[Gab05a] D. Gaboriau. Examples of groups that are measure equivalent to the free group. Ergodic Theory Dynam. Systems, 25(6):1809-1827, 2005.

[Gab05b] D. Gaboriau. Invariant percolation and harmonic Dirichlet functions. Geom. Funct. Anal., 15(5):1004-1051, 2005.

[Gab08] D. Gaboriau. Relative property (T) actions and trivial outer automorphism groups. preprint, 2008.

[Gab10a] D. Gaboriau. Approximations of equivalence relations. in preparation, 2010.

[Gab10b] D. Gaboriau. What is cost. preprint, 2010.

[Gef93] S. L. Gefter. Ergodic equivalence relation without outer automorphisms. Dopov./Dokl. Akad. Nauk Ukraïni, 11:25-27, 1993.

[Gef96] S. L. Gefter. Outer automorphism group of the ergodic equivalence relation generated by translations of dense subgroup of compact group on its homogeneous space. Publ. Res. Inst. Math. Sci., 32(3):517-538, 1996.

[GG88a] S. L. Gefter and V. Ya. Golodets. Fundamental groups for ergodic actions and actions with unit fundamental groups. Publ. Res. Inst. Math. Sci., 24(6):821847 (1989), 1988.

[GG88b] S. L. Gefter and V. Ya. Golodets. Fundamental groups for ergodic actions and actions with unit fundamental groups. Publ. Res. Inst. Math. Sci., 24(6):821847 (1989), 1988.

[Ghy95] É. Ghys. Topologie des feuilles génériques. Ann. of Math. (2), 141(2):387-422, 1995.

[GL09] D. Gaboriau and R. Lyons. A measurable-group-theoretic solution to von Neumann's problem. Invent. Math., 177(3):533-540, 2009.

[GP05] D. Gaboriau and S. Popa. An uncountable family of nonorbit equivalent actions of $\mathbf{F}_{n}$. J. Amer. Math. Soc., 18(3):547-559 (electronic), 2005. 
[GP07] T. Giordano and V. Pestov. Some extremely amenable groups related to operator algebras and ergodic theory. J. Inst. Math. Jussieu, 6(2):279-315, 2007.

[Gro93] M. Gromov. Asymptotic invariants of infinite groups. In Geometric group theory, Vol. 2 (Sussex, 1991), volume 182 of London Math. Soc. Lecture Note Ser., pages 1-295. Cambridge Univ. Press, Cambridge, 1993.

[Gro00] M. Gromov. Spaces and questions. Geom. Funct. Anal., (Special Volume, Part I):118-161, 2000. GAFA 2000 (Tel Aviv, 1999).

[Hjo05] G. Hjorth. A converse to Dye's theorem. Trans. Amer. Math. Soc., 357(8):3083-3103 (electronic), 2005.

[Hjo06] G. Hjorth. A lemma for cost attained. Ann. Pure Appl. Logic, 143(1-3):87-102, 2006 .

[HK05] G. Hjorth and A. S. Kechris. Rigidity theorems for actions of product groups and countable Borel equivalence relations. Mem. Amer. Math. Soc., 177(833):viii+109, 2005.

[HP99] O. Häggström and Y. Peres. Monotonicity of uniqueness for percolation on Cayley graphs: all infinite clusters are born simultaneously. Probab. Theory Related Fields, 113(2):273-285, 1999.

[Ioa07] A. Ioana. Orbit inequivalent actions for groups containing a copy of $\mathbf{F}_{2}$. preprint, 2007.

[Ioa08] A. Ioana. Cocycle superrigidity for profinite action of property (T) groups. Prépublication, 2008.

[Ioa09] A. Ioana. Relative property $(\mathrm{T})$ for the subequivalence relations induced by the action of $\mathrm{SL}_{2}(\mathbf{Z})$ on $\mathbf{T}^{\mathbf{2}}$. preprint, 2009.

[Ioa10] A. Ioana. $\mathrm{W}^{*}$-superrigidity for Bernoulli actions of property (T) groups, 2010.

[IPP08] A. Ioana, J. Peterson, and S. Popa. Amalgamated free products of weakly rigid factors and calculation of their symmetry groups. Acta Math., 200(1):85-153, 2008 .

[JKL02] S. Jackson, A. S. Kechris, and A. Louveau. Countable Borel equivalence relations. J. Math. Log., 2(1):1-80, 2002.

[JS87] Vaughan F. R. Jones and Klaus Schmidt. Asymptotically invariant sequences and approximate finiteness. Amer. J. Math., 109(1):91-114, 1987.

[Kaz67] D. A. Kazhdan. On the connection of the dual space of a group with the structure of its closed subgroups. Funkcional. Anal. i Priložen., 1:71-74, 1967. 
[Kec05] A. S. Kechris. Unitary representations and modular actions. Zap. Nauchn. Sem. S.-Peterburg. Otdel. Mat. Inst. Steklov. (POMI), 326(Teor. Predst. Din. Sist. Komb. i Algoritm. Metody. 13):97-144, 281-282, 2005.

[Kec10] A. S. Kechris. Global aspects of ergodic group actions, volume 160 of Mathematical Surveys and Monographs. A.M.S., 2010.

[Kid08a] Y. Kida. The mapping class group from the viewpoint of measure equivalence theory. Mem. Amer. Math. Soc., 196(916):viii+190, 2008.

[Kid08b] Y. Kida. Orbit equivalence rigidity for ergodic actions of the mapping class group. Geom. Dedicata, 131:99-109, 2008.

[Kid08c] Y. Kida. Outer automorphism groups of equivalence relations for mapping class group actions. J. Lond. Math. Soc. (2), 78(3):622-638, 2008.

[Kid09] Y. Kida. Rigidity in measure-theoretic group theory for amalgamated free products. Preprint, 2009.

[KM04] A. S. Kechris and B. D. Miller. Topics in orbit equivalence, volume 1852 of Lecture Notes in Mathematics. Springer-Verlag, Berlin, 2004.

[Koo31] B. O. Koopman. Hamiltonian systems and transformations in Hilbert space. Proceedings USA Academy, 17:315-318, 1931.

[KT08] A. S. Kechris and T. Tsankov. Amenable actions and almost invariant sets. Proc. Amer. Math. Soc., 136(2):687-697 (electronic), 2008.

[KT10] J. Kittrell and T. Tsankov. Topological properties of full groups. Ergodic Theory Dynam. Systems, to appear, 2010.

[Lev95] G. Levitt. On the cost of generating an equivalence relation. Ergodic Theory Dynam. Systems, 15(6):1173-1181, 1995.

[LP09] R. Lyons and Y. Peres. Probability on Trees and Networks. Cambridge University Press, In preparation, Cambridge, 2009.

[LPV08] R. Lyons, M. Pichot, and S. Vassout. Uniform non-amenability, cost, and the first $l^{2}$-Betti number. Groups Geom. Dyn., 2(4):595-617, 2008.

[LS99] R. Lyons and O. Schramm. Indistinguishability of percolation clusters. Ann. Probab., 27(4):1809-1836, 1999.

[LSW09] W. Lück, R. Sauer, and C. Wegner. L2-torsion, the measure-theoretic determinant conjecture, and uniform measure equivalence. preprint, 2009.

[Lüc94] W. Lück. Approximating $L^{2}$-invariants by their finite-dimensional analogues. Geom. Funct. Anal., 4(4):455-481, 1994. 
[Lüc02] W. Lück. $L^{2}$-invariants: theory and applications to geometry and $K$-theory, volume 44. Springer-Verlag, Berlin, 2002.

[Mar82] G. A. Margulis. Finitely-additive invariant measures on Euclidean spaces. Ergodic Theory Dynam. Systems, 2(3-4):383-396 (1983), 1982.

[MO10] N. Monod and N. Ozawa. The Dixmier problem, lamplighters and Burnside groups. J. Funct. Anal., 258(1):255-259, 2010.

[Mon06] N. Monod. An invitation to bounded cohomology. In International Congress of Mathematicians. Vol. II, pages 1183-1211. Eur. Math. Soc., Zürich, 2006.

[Moo82] C. C. Moore. Ergodic theory and von Neumann algebras. In Operator algebras and applications, Part 2 (Kingston, Ont., 1980), pages 179-226. Amer. Math. Soc., Providence, R.I., 1982.

[MS06] N. Monod and Y. Shalom. Orbit equivalence rigidity and bounded cohomology. Ann. of Math. (2), 164(3):825-878, 2006.

[MvN36] F. Murray and J. von Neumann. On rings of operators. Ann. of Math., II. Ser., 37:116-229, 1936.

[NR09] S. Neshveyev and S. Rustad. On the definition of $L^{2}$-Betti numbers of equivalence relations. Internat. J. Algebra Comput., 19(3):383-396, 2009.

[Ol'80] A. Ju. Ol'šanskiı̌. On the question of the existence of an invariant mean on a group. Uspekhi Mat. Nauk, 35(4(214)):199-200, 1980.

[OP08a] N. Ozawa and S. Popa. On a class of $\mathrm{II}_{1}$ factors with at most one cartan subalgebra. Ann. of Math., to appear, 2008.

[OP08b] N. Ozawa and S. Popa. On a class of $\mathrm{II}_{1}$ factors with at most one cartan subalgebra II, 2008.

[OW80] D. Ornstein and B. Weiss. Ergodic theory of amenable group actions. I. The Rohlin lemma. Bull. Amer. Math. Soc. (N.S.), 2(1):161-164, 1980.

[Oza04] N. Ozawa. Solid von Neumann algebras. Acta Math., 192(1):111-117, 2004.

[Oza06] N. Ozawa. A Kurosh-type theorem for type $\mathrm{II}_{1}$ factors. Int. Math. Res. Not., pages Art. ID 97560, 21, 2006.

[Oza09] N. Ozawa. An example of a solid von Neumann algebra. Hokkaido Math. J., 38(3):557-561, 2009.

[Pau99] F. Paulin. Propriétés asymptotiques des relations d'équivalences mesurées discrètes. Markov Process. Related Fields, 5(2):163-200, 1999.

[Pet09] J. Peterson. $L^{2}$-rigidity in von Neumann algebras. Invent. Math., 175(2):417433, 2009. 
[Pet10] J. Peterson. Examples of group actions which are virtually w*-superrigid, 2010.

[Pic07a] M. Pichot. Espaces mesurés singuliers fortement ergodiques (Étude métriquemesurée). Ann. Inst. Fourier (Grenoble), 57(1):1-43, 2007.

[Pic07b] M. Pichot. Sur la théorie spectrale des relations d'équivalence mesurées. $J$. Inst. Math. Jussieu, 6(3):453-500, 2007.

[Pop86] S. Popa. Correspondences. INCREST Preprint, 56, 1986.

[Pop06a] S. Popa. On a class of type $\mathrm{II}_{1}$ factors with Betti numbers invariants. Ann. of Math. (2), 163(3):809-899, 2006.

[Pop06b] S. Popa. Some computations of 1-cohomology groups and construction of nonorbit-equivalent actions. J. Inst. Math. Jussieu, 5(2):309-332, 2006.

[Pop06c] S. Popa. Strong rigidity of $\mathrm{II}_{1}$ factors arising from malleable actions of $w$-rigid groups. I. Invent. Math., 165(2):369-408, 2006.

[Pop06d] S. Popa. Strong rigidity of $\mathrm{II}_{1}$ factors arising from malleable actions of $w$-rigid groups. II. Invent. Math., 165(2):409-451, 2006.

[Pop07a] S. Popa. Cocycle and orbit equivalence superrigidity for malleable actions of $w$-rigid groups. Invent. Math., 170(2):243-295, 2007.

[Pop07b] S. Popa. Deformation and rigidity for group actions and von Neumann algebras. In I.C.M. Vol. I, pages 445-477. Eur. Math. Soc., Zürich, 2007.

[Pop08] S. Popa. On the superrigidity of malleable actions with spectral gap. J. Amer. Math. Soc., 21(4):981-1000, 2008.

[PS07] S. Popa and R. Sasyk. On the cohomology of Bernoulli actions. Ergodic Theory Dynam. Systems, 27(1):241-251, 2007.

[PSN00] I. Pak and T. Smirnova-Nagnibeda. On non-uniqueness of percolation on nonamenable Cayley graphs. C. R. Acad. Sci. Paris Sér. I Math., 330(6):495-500, 2000 .

[PV08a] S. Popa and S. Vaes. Actions of $\mathbf{F}_{\infty}$ whose $\mathrm{II}_{1}$ factors and orbit equivalence relations have prescribed fundamental group. preprint, 2008.

[PV08b] S. Popa and S. Vaes. Cocycle and orbit superrigidity for lattices in $\operatorname{SL}(n, \mathbb{R})$ acting on homogeneous spaces, 2008.

[PV08c] S. Popa and S. Vaes. On the fundamental group of $\mathrm{II}_{1}$ factors and equivalence relations arising from group actions, 2008.

[PV08d] S. Popa and S. Vaes. Strong rigidity of generalized Bernoulli actions and computations of their symmetry groups. Adv. Math., 217(2):833-872, 2008. 
[PV09a] M. Pichot and S. Vassout. Le coût est un invariant isopérimétrique. to appear in Journal of Non comm. Geom., 2009.

[PV09b] S. Popa and S. Vaes. Group measure space decomposition of $\mathrm{II}_{1}$ factors and $\mathrm{W}^{*}$-superrigidity, 2009.

[Sak09a] H. Sako. The class $S$ as an ME invariant. Int. Math. Res. Not. IMRN, (15):2749-2759, 2009.

[Sak09b] H. Sako. Measure equivalence rigidity and bi-exactness of groups. J. Funct. Anal., 257(10):3167-3202, 2009.

[Sau05] R. Sauer. $L^{2}$-Betti numbers of discrete measured groupoids. Internat. J. Algebra Comput., 15(5-6):1169-1188, 2005.

[Sau09] R. Sauer. Amenable covers, volume and $L^{2}$-Betti numbers of aspherical manifolds. J. Reine Angew. Math., 636:47-92, 2009.

[Sch80] K. Schmidt. Asymptotically invariant sequences and an action of $\mathrm{SL}(2, \mathbf{Z})$ on the 2-sphere. Israel J. Math., 37(3):193-208, 1980.

[Sch81] K. Schmidt. Amenability, Kazhdan's property T, strong ergodicity and invariant means for ergodic group-actions. Ergodic Theory Dynam. Systems, 1(2):223-236, 1981.

[Ser77] J.-P. Serre. Arbres, amalgames, SL 2 , volume 46 of Astérisque. S.M.F., 1977.

[Sha04] Y. Shalom. Harmonic analysis, cohomology, and the large-scale geometry of amenable groups. Acta Math., 192(2):119-185, 2004.

[Sha05] Y. Shalom. Measurable group theory. In European Congress of Mathematics, pages 391-423. Eur. Math. Soc., Zürich, 2005.

[Sin55] I. M. Singer. Automorphisms of finite factors. Amer. J. Math., 77:117-133, 1955.

[ST07] R. Sauer and A. Thom. A spectral sequence to compute $L^{2}$-betti numbers of groups and groupoids. 2007.

[Tho08] A. Thom. $L^{2}$-invariants and rank metric. In $C^{*}$-algebras and elliptic theory II, Trends Math., pages 267-280. Birkhäuser, Basel, 2008.

[Tho09] A. Thom. Low degree bounded cohomology and $L^{2}$-invariants for negatively curved groups. Groups Geom. Dyn., 3(2):343-358, 2009.

[Tör06] A. Törnquist. Orbit equivalence and actions of $\mathbf{F}_{\mathbf{n}}$. J. Symbolic Logic, 71(1):265-282, 2006. 
[Vae07] S. Vaes. Rigidity results for Bernoulli actions and their von Neumann algebras (after Sorin Popa). Number 311, pages Exp. No. 961, viii, 237-294. S.M.F., 2007. Séminaire Bourbaki. Vol. 2005/2006.

[Vae10] S. Vaes. Rigidity for von neumann algebras and their invariants. Proceedings of the International Congress of Mathematicians, Hyderabad, India, 2010. Proceedings of the International Congress of Mathematicians (Hyderabad, India, 2010), Vol. III, pp. 1624-1650 Hindustan Book Agency, 2010.

[Val05] A. Valette. Group pairs with property (T), from arithmetic lattices. Geom. Dedicata, 112:183-196, 2005.

[vN29] J. von Neumann. Zur allgemeinen theorie des maßes. Fund. Math., 13:73-116, 1929.

[Zim84] R. J. Zimmer. Ergodic theory and semisimple groups, volume 81 of Monographs in Mathematics. Birkhäuser Verlag, Basel, 1984.

Damien Gaboriau

Unité de Mathématiques Pures et Appliquées

Université de Lyon, CNRS, Ens-Lyon

46 , allée d'Italie

69364 Lyon cedex 7, France

gaboriau@umpa.ens-lyon.fr 